\title{
Shock waves induce changes in meningiomas of the brain
}

\author{
B. J. Sudhir ${ }^{1, *}$, Anita Mahadevan ${ }^{2}$ and K. P. J. Reddy ${ }^{3}$ \\ ${ }^{1}$ Department of Neurosurgery, Sree Chitra Tirunal Institute for Medical Sciences and Technology, Thiruvananthapuram 695011 , India \\ ${ }^{2}$ Department of Neuropathology, National Institute of Mental health and Neuro Sciences, Bengaluru 560029 , India \\ ${ }^{3}$ Department of Aerospace Engineering, Indian Institute of Science, Bengaluru 560 012, India
}

\begin{abstract}
The effect of shock waves on biological systems has been extensively researched for its traumatic effects. Few research groups have attempted and successfully harnessed the power of shock waves for therapeutic benefits. In neurosurgical applications, shock waves have been explored as a brain dissection tool. However, the difficulties of controlling the effects of shock waves preclude the safe use of shock waves in neurosurgery. The present study focuses on understanding the effects of shock waves on meningiomas, tumours that arise from the protective layers of the brain. Freshly excised meningioma specimens, subjected to shock waves generated manually using a Reddy tube, were examined.
\end{abstract}

Keywords: Meningioma, neurosurgery, Reddy tube, shock tube, shock waves.

BRAIN tumours vary in consistency depending on their tissue of origin and tumour tissue framework. They vary from cysts to hard bony tumours. Meningiomas are tumours that arise from the meninges, the protective membranous layer surrounding the brain and spinal cord. They are usually firm, sometimes soft and rarely hard. At diagnosis they range in size from small tumours that come to attention by symptoms such as seizures to large bulky tumours that remain silently asymptomatic. These tumours are usually firm in consistency, a feature depending on the tissue characteristics of a dense fibrous framework. It is rare for these tumours to be cystic or calcified. The consistency of meningiomas is also determined by its vascularity. Surgical principles for safe tumour removal include de-bulking the tumour piecemeal to reduce it to a shell and then dissecting it meticulously from the surrounding brain.

Meningioma surgery has influenced the history and evolution of neurosurgery over centuries. Technological advancements inducted into neurosurgical practice made neurosurgery easier and safer for patients. In the eighteenth century, surgical explorations, caustic lime application and extirpation of meningioma by the surgeon's fingers, resulted in death due to infection and bleeding ${ }^{1}$. The introduction of electro-cautery in surgery enabled

*For correspondence. (e-mail: bjs@sctimst.ac.in) haemostasis and improved survival following surgery for meningioma ${ }^{2,3}$. Bipolar electrocautery, monopolar electrocautery with ring-tip remained the adjunct for tumour de-bulking. Soft tumours yield to gentle manipulation and suction, while firm and hard tumours are cut with a surgical blade. The introduction of the cavitron ultrasonic suction aspirator (CUSA, Söring $₫ /$ Stryker $\AA$ Sonopet) further eased the process of de-bulking ${ }^{4-6}$. Despite improvements in CUSA technology such as the introduction of the shear wave technology, neurosurgeons still face difficulties in pulverizing firm and hard meningiomas ${ }^{7}$.

Shock tubes have been used for a variety of applications. They have traditionally been devised by engineers to understand shock waves, simulate explosions and research mitigation techniques ${ }^{8}$. Applications include heat and gas flow studies in jet engine design, space shuttle re-entry simulation, and molecular chemical kinetics studies $^{9-11}$. The possibility of using controlled shockwaves to disintegrate firm-consistency meningiomas formed the basis of our experiments. Manually operated piston-driven shock tube, capable of delivering Mach 2 shock waves was used for the experiments ${ }^{12}$.

\section{Methodology}

The shock tube was devised using a medical syringe as the driver segment (Figure $1 a$ ). A plastic chamber was designed to hold a thin plastic film membrane interposed between the syringe and driven-segment (Figure $1 b$ ). For the driven segment a plastic tube of internal diameter $2 \mathrm{~mm}$ was used. The piston was manually driven to rupture the plastic membrane to generate a shock-wave in the driven section at the end of which the tissue specimen was placed. A specially designed steel chamber was used for holding the tissue and to prevent dislodgement of the tissue during shock wave exposure (Figure $1 c$ ). The technical details and performance parameters of the Reddy tube have been previously described ${ }^{12}$.

The study protocol was approved by the Institute Ethics Committee. Four patients with a diagnosis of large intracranial meningioma were included in the study. The availability of sufficient tissue sample for an accurate histopathological diagnosis was ensured. The specimen was divided into two pieces. The average size of the 


\section{RESEARCH ARTICLES}

divided specimen was $2.5 \mathrm{~cm}$. One part of the divided tissue specimen was subjected to shock wave exposure using a Reddy tube. The other part was labelled as control. The shock-wave exposed specimen and the control specimen were immediately fixed in formalin and subsequently subjected to histopathological examination. Gross and microscopic examination studies were performed and observations recorded.

\section{Results}

Four sets of samples (experimental and control) were examined for their gross and microscopic features. The consistency of these tumours at surgery was described as firm by the operating surgeon. These tumours had to be cut with a surgical blade for de-bulking during surgery.

On gross examination, two samples subjected to shockwaves showed evidence of changes attributable to shockwaves. One specimen showed linear zones of congestion close to the surface while the other specimen showed a pale glistening myxoid area running along the periphery close to the surface.

Using high power microscopy, two of the specimens were histologically identified to be fibroblastic meningiomas, one was an atypical meningioma and the fourth specimen was an angiomatous meningioma. The description of microscopic features in the four specimens subjected to shock-wave is as follows.

\section{Specimen 1}

Histopathological diagnosis: Fibroblastic meningioma Grade I.

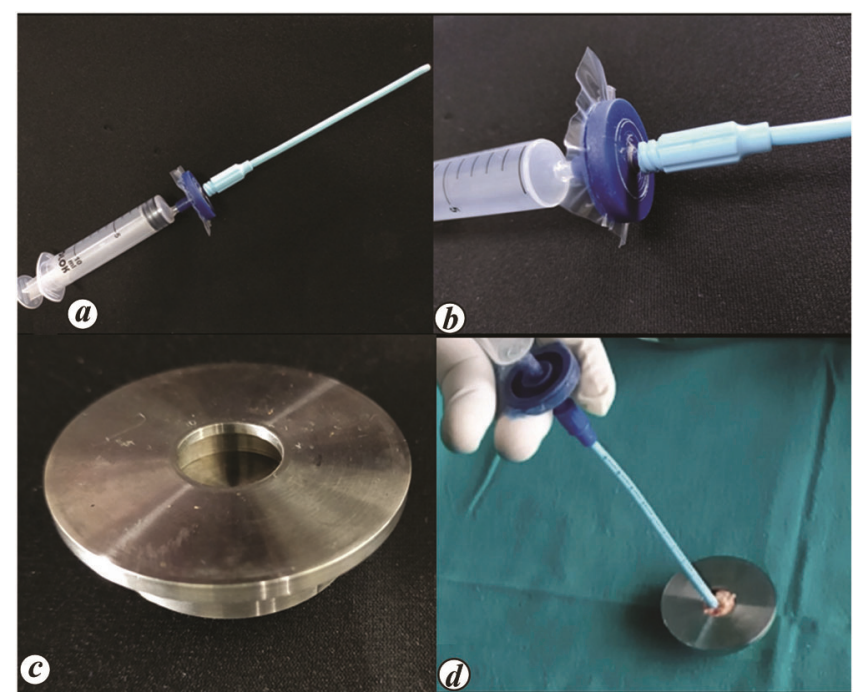

Figure 1. $\boldsymbol{a}$, The Reddy tube crafted using a medical syringe. $\boldsymbol{b}$, The casing interposed between the syringe and the driven section tube holds a plastic film diaphragm. $c$, Specially designed tissue holder with a rimmed cavity that prevents dislodgement of the tissue during shock wave exposure. $\boldsymbol{d}$, The manual application of shock wave on meningioma specimen using the Reddy tube.
Histopathological features in test specimen: Zones of haemorrhage were noted close to the surface, both as a thin film capping tumour at interface with dura and disrupting the tumour beneath (to a depth of $\sim 3 \mathrm{~mm}$ ) with oedema and vacuolation of stroma focally. The large thick-walled meningeal vessels were intact with thick muscle coat showing spasm. The smaller venules within the tumour were disrupted with diapedesis of RBCs into the adjacent tumour. Focal cavitation with cystic space was seen in tumour stroma with surrounding vacuolation and haemorrhage. The tumour adjacent to the haemorrhage and cavitation did not show morphological alterations. The nuclear character and cytoplasm were well preserved including presence of nuclear grooves. An intriguing finding noted was a mild degenerative nuclear atypia; however, no increase in mitosis or necrosis was identified (Figure 2).

\section{Specimen 2}

Histopathological diagnosis: Fibroblastic meningioma Grade I.

Histopathological features in test specimen: Dense stromal and vascular hyalinization and small foci of perivascular coagulative necrosis were observed. Within these zones, the tumour cells appeared rounded with nuclear smudging and karyorrhexis. Along the periphery of the tumour, multiple rounded scarred foci of varying sizes were observed having seams of thick collagen appearing to encircle empty vascular channels. Most had no remnant endothelial lining (Figure 3).

\section{Specimen 3}

Histopathological diagnosis: Atypical meningioma Grade II.

Histopathological features in test specimen: The specimen showed a large geographic focus of scarring. In this zone thin strands of delicate collagen were seen traversing the scar. The tumour cells appear to be dissolved out except for strands of entrapped tumour cells traversing the scar. The stroma was loose, oedematous and vacuolated. The vessels within the scar were noted to be thin walled and did not show much collagen except in the core of the scar where thin dilated veins were entrapped in collar of collagen. Shrinkage of the nucleus with loss of nucleoli was noted immediately subjacent to the scar. Higher mitotic activity was observed. The lesioned area showed more prominent shrinkage and periphery shows intact viable tumour (Figure 4). 


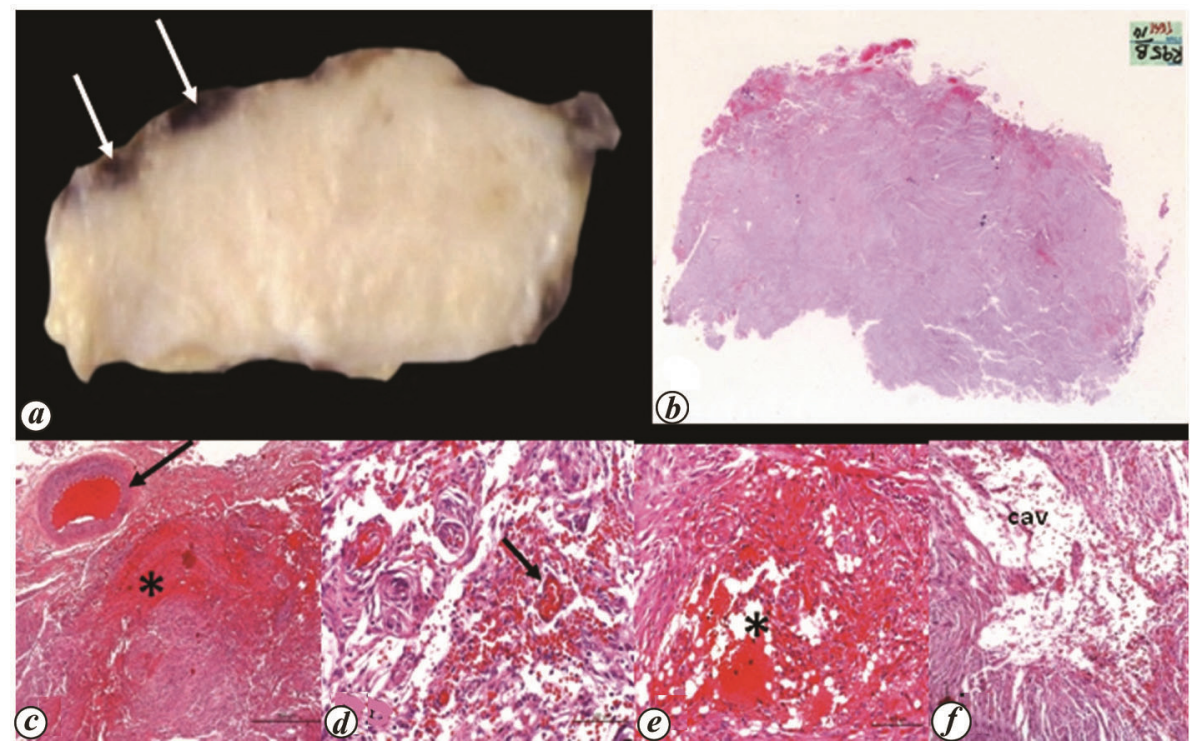

Figure 2. $\boldsymbol{a}$, Surface shows small foci of haemorrhage in gross specimen; $\boldsymbol{b}$, On microscopy haemorrhage is seen only close to surface; $c$, Haemorrhage along surface of tumour $(*)$ with large meningeal vessels (arrow) showing spasm but no rupture; $\boldsymbol{d}$, Intra-parenchymal venules (arrow) show rupture; $\boldsymbol{e}$, Zones of intra-tumoural haemorrhage; $\boldsymbol{f}$, Small cystic cavitation within the tumour.

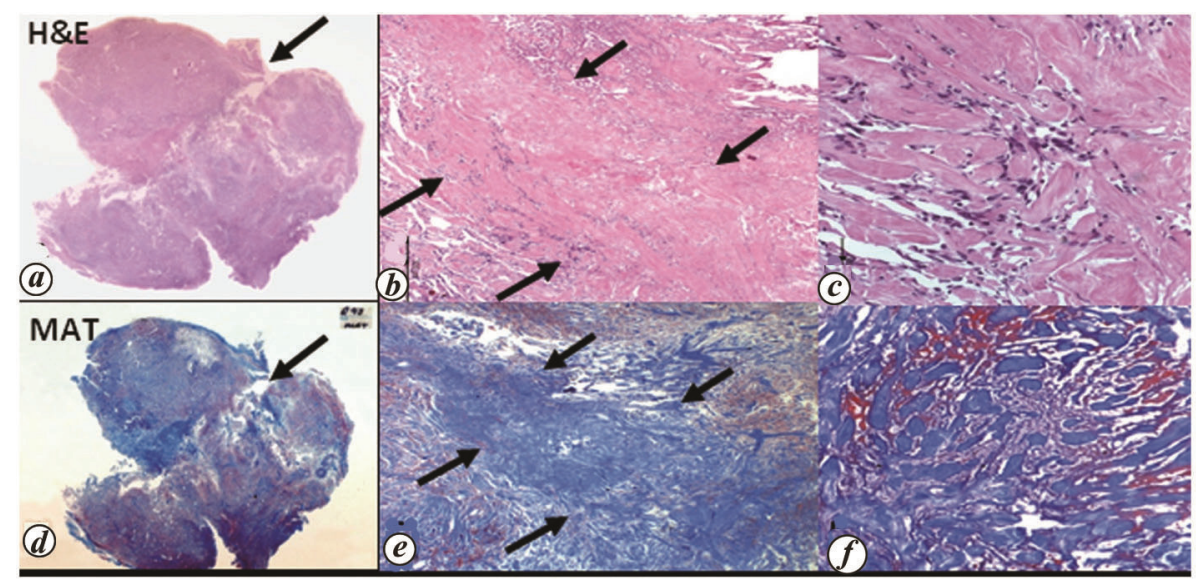

Figure 3. $\boldsymbol{a}, \boldsymbol{d}$, Whole mount view of tumour shows dense fibrosis (arrow) along surface; $\boldsymbol{b}, \boldsymbol{c}$, Closeup view of large zones of scarring in tumour (arrows); $\boldsymbol{e}, \boldsymbol{f}$, Thick bands of collagen replacing tumour (e) seen as blue bands on Masson trichrome (MAT) with few remnant tumour cells entrapped $(\boldsymbol{f})$.

\section{Specimen 4}

Histopathological diagnosis: Angiomatous meningioma Grade I.

Histopathological features in test specimen: Loss of microcystic areas in the stroma was observed. The nodular aggregates of the tumour appeared coalescent with inter-nodular areas showing collapse of stroma causing numerous small blood vessels to aggregate. The large hyalinized vessels appeared less conspicuous. Some of the nodules of the tumour cells showed central cavitation. Along the periphery, the stroma was loose with aggregation of hyalinized vessels (Figure 5).
To summarize, fibroblastic meningiomas subjected to shock-wave revealed haemorrhage, vascular congestion, oedema, vacuolation, coagulative necrosis and prominent vascular/stromal hyalinization. The atypical meningioma specimen showed large zone of scarring with dissolution of tumour cells. Loss of microcystic spaces, cavitation of tumour, aggregation and arterialization of small blood vessels were noted in the Angiomatous meningioma with microcystic change.

\section{Discussion}

Shock waves have been used in a plethora of medical fields. Currently, shock wave as a therapeutic modality 


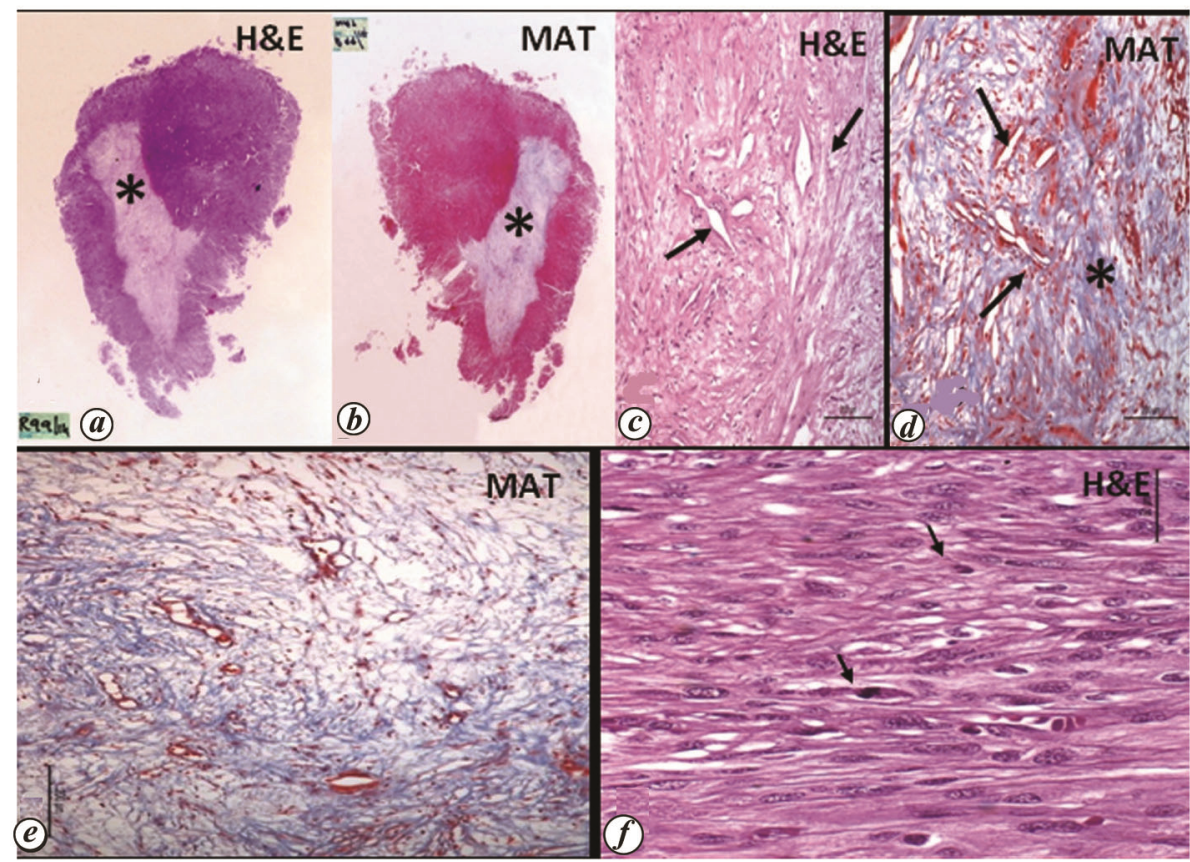

Figure 4. Large central scar $\left(^{*}\right)$ is seen $(\boldsymbol{a}, \boldsymbol{b})$ with core showing thin veins $(\boldsymbol{c}$, arrow) entrapped in collagen and loose matrix $(\boldsymbol{d}, *)$. No tumour cells are seen within the scar $(\boldsymbol{e})$. Adjacent to scar, tumour showed shrinkage of tumour cells with more frequent mitosis $(\boldsymbol{f})$. (H\&E, Hematoxylin and eosin stain; MAT, Masson's trichrome stain).

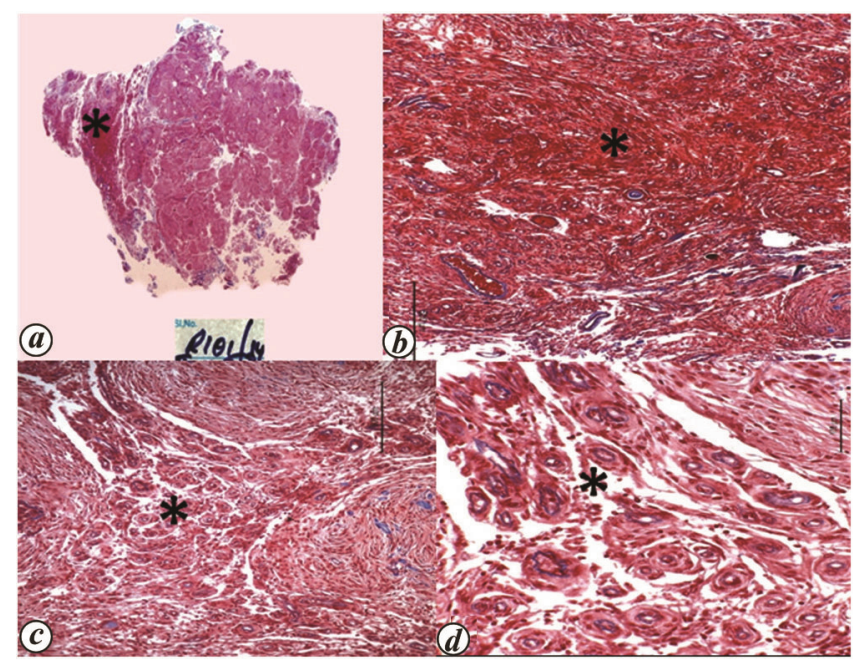

Figure 5. Tumour shows intratumoral haemorrhage $\left(\boldsymbol{a}^{*}, \boldsymbol{b}^{*}\right)$. There is loss of microcystic change, collapse of nodules with shrinkage of vessels forming a leash between the nodules $(*, c, d)$.

has been approved only for limited indications, while several applications in medicine have been explored. Extracorporeal shock wave lithotripsy (ESWL) is used in pulverizing renal, gallbladder and pancreatic calculi ${ }^{13-15}$. Shock waves have been found useful in the treatment of orthopaedic conditions, tapping the osteo-inductive and other potential biological effects of shock waves ${ }^{16}$. Tendinopathies, plantar fasciitis and bone non-union have been treated successfully.

The use of shock waves in head and neck region of the human body, particularly the brain, has certain limita- tions. Salivary gland calculi have been successfully treated with shock waves ${ }^{17}$. Certain factors restrict the use of shock waves in the vicinity of the brain. The brain is a soft organ and the transmission of shock wave applied on the brain is unpredictable and difficult to control. Thus, restricting the effect of shock wave to a target area would be difficult. Secondly, the hardware and technique to deliver a shock wave of predicted intensity and propagation to target areas in the brain where neural tissue and blood vessels are anatomically placed intricately is lacking $^{18}$. Experimental studies on clot disintegration in stroke using shock wave induced liquid jets have not yet evolved into clinical practice ${ }^{19,20}$. Shock wave technology has also been used for gene delivery into cells ${ }^{21}$. Using similar principles, shock wave-driven drug delivery systems have been developed ${ }^{22}$.

Underwater shock wave generated using pulsed Ho : YAG laser beam irradiation in water have been experimentally studied on animal brains for brain tissue dissection and endoscopic ventriculostomy ${ }^{23}$. Shock wave mitigation by using Gore-Tex dural substitute has also been studied ${ }^{24}$. Complex neuro-anatomy, acoustic properties of the brain and technical issues with focusing shock waves at the exact pathological site where the effect is expected preclude the safe use of shock wave as a therapeutic modality in neurosurgery. However, shock waves continue to evoke interest among neuroscience researchers. In the treatment of spasticity, shock wave application has been found to be beneficial ${ }^{25,26}$.

Acts of terrorism and accidental explosions expose soldiers and civilians to blast generated shock waves. 
Blast induced neuro-trauma (BINT) is a less-understood phenomenon. Researchers have attempted to replicate BINT using animal studies in free-field blasts and shocktube generated shock waves ${ }^{27,28}$. Although results of these studies provide an insight into the destructive effects of shock waves on the brain, controlled experiments in an attempt to harness the power of shock waves for neurosurgical applications are rare.

Tumours arising from the meninges, the protective layers of the brain termed as meningiomas vary in consistency from being soft at one end of the spectrum to being hard and calcified at the other. Soft tumours are extirpated with ease using dissection and suction techniques. Firm tumours are usually removed using a cavitron ultrasonic suction aspirator (CUSA, Söring ${ }^{\circledR} /$ Stryker ${ }^{\circledR}$ Sonopet). Hard tumours are extremely difficult to be de-bulked. The neurosurgeon uses a sharp scalpel blade to surgically cut sections of the tumour in situ, before dissecting the tumour from the brain. This procedure is risky especially in deep surgical fields with vital neurovascular structures in the vicinity of the tumour. In this scenario, the use of controlled shock waves to pulverize the tumour and soften it in situ was contemplated. Against this backdrop, this in-vitro study was done in freshly excised meningioma specimens using a shocktube.

The shock waves produced unique findings in the meningioma specimens. Congestion and myxoid changes were identified on gross examination in two specimens. Focal oedema and vacuolation, cavitation with cystic changes, coagulative necrosis and hyalinization were noted to a depth of $3 \mathrm{~mm}$ in the tumour specimen subjected to shock wave. The venules within the tumour exhibited disruption with diapedesis of RBCs into the adjacent tumour.

The changes inflicted by the shock waves were confined to the superficial layers of the tumour. There was no significant change in consistency of the areas facing the shock wave front. Dissolution of tumour cells, oedema, vacuolation, cavitation, coagulative necrosis could be associated with softening of the tumour. These changes were microscopic and observed in a small portion of the tumour facing the pressure wave. The microscopic changes noted in the tumour suggest a promising application of shock wave in tumour disintegration in neurosurgery. However, issues concerning the expected damage to underlying brain by the unrestricted passage of shockwaves to subjacent brain, limit the clinical use of shockwave based technology in brain tumour surgery.

\section{Conclusion}

Shock waves produced gross and unique microscopic changes in meningiomas. The study provides an insight into the effect of shock-waves on meningiomas. The safety concerns of shock-waves used to produce changes in meningiomas precludes its use in neurosurgical procedures. Future research should be focused on techniques to control the depth of penetration of shock wave, shock wave mitigation techniques and improve the safety profile for use in neurosurgery.

\section{Declarations}

Funding: The authors have not received funding from any source for support of the research.

Conflict of interest statement: The authors declare no conflict of interest.

Ethics approval: The study was approved by the Institute Ethics Committee at Sree Chitra Tirunal Institute for Medical Sciences and Technology, Thiruvananthapuram.

Consent to participate: Written informed consent was obtained from the patient/guardian to participate in the study.

Consent for publication: Approval was obtained for publication of anonymised clinical data and images.

1. Al-Rodhan, N. R. F. and Laws, E. R., Meningioma: A historical study of the tumor and its surgical management. Neurosurgery, 1990, 26, 832-847.

2. Goldwyn, R. M., Bovie: The man and the machine. Ann. Plastic Surg., 1979, 2, 135-153.

3. Bulsara, K. R., Sukhla, S. and Nimjee, S. M., History of bipolar coagulation. Neurosurg. Rev., 2006, 29, 93-96; discussion 96.

4. Fasano, V. A., Zeme, S., Frego, L. and Gunetti, R., Ultrasonic aspiration in the surgical treatment of intracranial tumors. $\mathrm{J}$. Neurosurg. Sci., 1981, 25, 35-40.

5. Goldsmith, M. F., Ultrasonic device wins neurosurgeon praise. JAMA, 1983, 250, 3270-3270

6. Epstein, F. J. and Farmer, J. P., Trends in surgery: laser surgery, use of the cavitron, and debulking surgery. Neurol. Clin., 1991, 9, 307-315.

7. Kurokawa, Y., Ishiguro, M. and Kurokawa, T. A., Giant true ossified meningioma removed with surgical ultrasonic aspirator with shear wave technology. Clin. Surg., 2017, 2, 1829.

8. Ma, S., Zhang, X., Lian, Y. and Zhou, X., Simulation of high explosive explosion using adaptive material point method. CMES CMES-Comp Model Eng., 2009, 39(2), 101-123.

9. Vasu, S. S., Davidson, D. F. and Hanson, R. K., Jet fuel ignition delay times: Shock tube experiments over wide conditions and surrogate model predictions. Combust. Flame, 2008, 152, 125143.

10. Horvath, T. J., Cagle, M. F., Grinstead, J. H. and Gibson, D. M., Remote observations of reentering spacecraft including the space shuttle orbiter. In 2013 IEEE Aerospace Conference, 2013, pp. $1-15$.

11. Lin, S. and Fyfe, W. I., Low-density shock tube for chemical kinetics studies. Phys. Fluids, 1961, 4, 238-249.

12. Reddy, K. P. J. and Sharath, N., Manually operated piston-driven shock tube. Curr. Sci., 2013, 104, 5.

13. Nightingale, S. L. and Young, F. E., Marketing approval for the lithotripter. Isr. J. Med. Sci., 1986, 22, 519-523. 
14. Sackmann, M., Pauletzki, J., Sauerbruch, T., Holl, J., Schelling, G. and Paumgartner, G., The Munich Gallbladder Lithotripsy Study. Results of the first 5 years with 711 patients. Ann. Intern. Med., 1991, 114, 290-296.

15. Sauerbruch, T., Holl, J., Sackmann, M., Werner, R., Wotzka, R. and Paumgartner, G., Disintegration of a pancreatic duct stone with extracorporeal shock waves in a patient with chronic pancreatitis. Endoscopy, 1987, 19, 207-208.

16. Moya, D., Ramón, S., Schaden, W., Wang, C.-J., Guiloff, L. and Cheng, J.-H., The role of extracorporeal shockwave treatment in musculoskeletal disorders. JBJS, 2018, 100, 251-263.

17. Iro, H. et al., Shockwave lithotripsy of salivary duct stones. Lancet, 1992, 339, 1333-1336.

18. Nakagawa, A. et al., Application of shock waves as a treatment modality in the vicinity of the brain and skull. J. Neurosurg., 2003, 99, 156-162.

19. Takayama, K. and Ohtani, K., Applications of shock wave research to medicine. WIT Trans. Modell. Simulat., 2005, 41, 9.

20. Kodama, T., Takayama, K. and Uenohara, H., A new technology for revascularization of cerebral embolism using liquid jet impact. Phys. Med. Biol., 1997, 42, 2355-2367.

21. Klein, R. M., Wolf, E. D., Wu, R. and Sanford, J. C., Highvelocity microprojectiles for delivering nucleic acids into living cells. Biotechnology, 1992, 24, 384-386.

22. Battula, N., Menezes, V. and Hosseini, H., A miniature shock wave driven micro-jet injector for needle-free vaccine/drug delivery. Biotechnol. Bioeng., 2016, 113, 2507-2512.
23. Tominaga, T. et al., Application of underwater shock wave and laserinduced liquid jet to neurosurgery. Shock Waves, 2006, 15, 55.

24. Nakagawa, A., Kusaka, Y., Hirano, T., Saito, T., Shirane, R., Takayama, K. and Yoshimoto, T., Application of shock waves as a treatment modality in the vicinity of the brain and skull. J. Neurosurg., 2003, 99(1), 156-162.

25. Guo, P., Gao, F., Zhao, T., Sun, W., Wang, B. and Li, Z., Positive effects of extracorporeal shock wave therapy on spasticity in poststroke patients: a meta-analysis. J. Stroke Cerebrovasc. Dis., 2017, 26, 2470-2476.

26. Oh, J. H., Park, H. D., Han, S. H., Shim, G. Y. and Choi, K. Y., Duration of treatment effect of extracorporeal shock wave on spasticity and subgroup - analysis according to number of shocks and application site: a meta-analysis. Ann. Rehabil. Med., 2019, 43, 163-177.

27. Rubovitch, V. et al., A mouse model of blast-induced mild traumatic brain injury. Exp. Neurol., 2011, 232, 280-289.

28. Bhat, D. I., Shukla, D., Mahadevan, A., Sharath, N. and Reddy, K. P. J., Validation of a blast induced neurotrauma model using modified Reddy tube in rats: A pilot study. Indian J. Neurotrauma, 2014, 11, 91-96.

Received 25 November 2020; accepted 6 March 2021

doi: $10.18520 / \mathrm{cs} / \mathrm{v} 120 / \mathrm{i} 10 / 1587-1592$ 